\title{
Educação física e diabetes: prevenção e tratamento
}

\author{
Guilherme V. Giorelli, ${ }^{1 \star}$ Fernanda M. Santos, ${ }^{1}$ Leonardo H. Portes $^{1}$
}

\begin{abstract}
Resumo
O objetivo deste estudo é revisar a literatura sobre a avaliação pré-intervenção e prescrição do exercício físico para pacientes diabéticos, além das principais recomendações para uma prática segura e eficaz. Por muitos anos, o exercício físico tem sido considerado, juntamente com a dieta e a medicação, um componente fundamental no tratamento e prevenção do diabetes. O exercício, através da contração muscular, permite a captação de glicose, colaborando no controle glicêmico do diabético, que, por sua vez, reflete na prevenção de complicações. Tanto os exercícios aeróbios quanto os exercícios resistidos diminuem a glicemia, a HbA1c e melhoram o perfil lipídico, parâmetros estes essenciais para um bom controle metabólico do paciente diabético. No entanto, antes que o diabético comece efetivamente a prática de exercício, é necessária uma pré-avaliação, para que sua condição clínica e metabólica seja analisada, e que o risco não seja maior que o benefício. Sendo assim, os pacientes diabéticos devem ser estimulados a praticar o exercício físico, visando um bom estado de saúde e bem-estar. Com base nesta revisão, podemos dizer que o exercicio é uma das estratégias que podem estimular a melhora da saúde do paciente diabético de forma global, pois apresenta viabilidade na prática clínica e não necessita de altos custos. É importante dizer que a prescrição deve ser individualizada e realizada por um profissional capacitado, além de respeitadas as recomendações para esta população especial, proporcionando uma prática segura e eficaz.
\end{abstract}

Descritores: Diabetes mellitus; Exercício; Prevenção \& controle; Prescriçõos.

\section{Abstract \\ Physical education and diabetes: prevention and treatment}

The aim of this study is to review the literature on pre-intervention evaluation and prescription of exercise for patients with diabetes, the key recommendations for a safe and effective practice. For many years, exercise has been considered, along with diet and medication, a key component in the treatment and prevention of diabetes. The exercise by muscle contraction, allows glucose uptake, collaborating on glycemic control, which in turn reflects on the prevention of complications. Both aerobic exercises as resistance exercises reduce blood glucose and HbA1c and improves the lipid profile, these parameters are essential for a good metabolic control in diabetic patients. However, a pre-assessment before the patient effectively begin the practice of exercise is necessary so that their clinical and metabolic condition is evaluated,
1. Serviço de Diabetes e Metabologia. Policlínica Piquet Carneiro. Universidade do Estado do Rio de Janeiro. Rio de Janeiro, RJ, Brasil.

*Endereço para correspondência:

Ambulatório de Diabetes e Metabologia, Policlínica Piquet Carneiro Av. Marechal Rondon, 381, 3음 andar

Rio de Janeiro, RJ, Brasil. CEP: 20950-000.

E-mail: guilherme@giorelli.com.br

Revista HUPE, Rio de Janeiro, 2015;14(4):54-58

doi: $10.12957 /$ rhupe.2015.20058

Recebido em 28/11/2014. Aprovado em 22/12/2014.

where the risk is no greater than the benefit. Thus, diabetic patients should be encouraged to practice physical exercise aiming at a good state of health and well-being. Based on this review, we can say that exercise is one of the strategies that can stimulate the improvement of health of patients with diabetes globally, presenting viability in clinical practice and that do not require high costs. Importantly, the prescription must be individualized and performed by a trained professional, respecting the recommendations for this special population by providing a safe and effective practice.

Keywords: Diabetes mellitus; Exercise; Prevention \& control; Prescriptions.

\section{Resumen}

\section{Educación física y diabetes: prevención y trata- miento}

El objetivo de este estudio es revisar la literatura sobre la evaluación previa a la intervención y prescripción de ejercicio físico para pacientes diabéticos, además de las principales recomendaciones para una práctica segura y eficaz. Durante muchos años, el ejercicio físico se ha considerado, junto con la dieta y la medicación, un componente esencial en el tratamiento y prevención de la diabetes. El ejercicio, a través de la contracción muscular, permite la captación de glucosa, colaborando en el control glucémico del diabético, lo que a su vez incide en la prevención de complicaciones. Tanto los ejercicios aeróbicos como ejercicios de resistencia reducen la glucosa en sangre, la HbA1c y mejoran el perfil lipídico, parámetros esenciales para un buen control metabólico del paciente diabético. Sin embargo, antes que el diabético comience efectivamente la práctica de ejercicio, se requiere una evaluación previa, para que su condición clínica y metabólica 
sea analizada, y que el riesgo no sea mayor que el beneficio. Por lo tanto, los pacientes diabéticos deben ser alentados a practicar ejercicio físico, con vistas a un buen estado de salud y bienestar. Sobre la base de esta revisión, podemos decir que el ejercicio es una de las estrategias que puede mejorar la salud de los pacientes diabéticos de forma global, es viable en la práctica clínica y no requiere de altos costos. Es importante destacar

\section{Introdução}

Pesquisas realizadas nas últimas décadas demonstram claramente que o exercício físico aumenta a captação de glicose através da contração muscular esquelética.Sendo assim, o exercício hoje é considerado um dos pilares tanto da prevenção do diabetes tipo 2 como no tratamento do diabetes 1 e 2 .

O exercício físico age diretamente sobre a resistência à insulina, além de aumentar a capilarização nas células musculares esqueléticas. Além da influência positiva no controle do diabetes, o exercício é um coadjuvante no tratamento de outras comorbidades que podem estar combinadas ao diabetes, como doenças cardíacas, dislipidemia e obesidade. ${ }^{1}$

Entre essas comorbidades, as doenças cardíacas merecem atenção especial, pois são as principais causas de morte relacionadas ao diabetes. O exercício, ao prevenir tais doenças e aprimorar a capacidade cardiorrespiratória, pode gerar benefícios adicionais, tais como: melhora do perfil lipídico, disponibilidade de glicose, aprimoramento da função endotelial e manutenção ou perda de massa corporal total. ${ }^{2}$

Pelo caráter multissistêmico e agressivo do diabetes, recomendam-se avaliações periódicas do diabético que se exercita, procurando minimizar complicações, as quais deverão contemplar os principais sistemas acometidos, incluindo avaliações cardíaca, vascular, autonômica, renal e oftalmológica. ${ }^{1}$

Assim, o objetivo deste estudo é revisar a literatura sobre a avaliação pré-intervenção, prescrição do exercício físico para esta população e as principais recomendações para uma prática segura e eficaz.

\section{Avaliação pré-exercício}

Idealmente, antes de iniciar um programa de exercício físico intenso, a maioria dos diabéticos deve passar por avaliação médica. Entretanto, esta avaliação médica não deve ser uniforme em todos os pacientes diabéticos, pois cada nível de exercício físico tem uma exigência. ${ }^{3}$

Algumas informações são de grande importância para o profissional de educação física, visando otimizar a prescrição do exercício no paciente diabético. Dados que la prescripción debe ser individualizada y realizada por un profesional calificado, así como el cumplimiento de las recomendaciones para esta población especial, proporcionando una práctica segura y eficaz.

Palabras clave: Diabetes mellitus; Ejercicio; Prevención y control; Prescripciones.

de exame físico como peso, frequência cardíaca e pressão arterial em repouso são indispensáveis. Informações médicas, como presença de edema e anormalidades dos pulsos arteriais de membros inferiores, avaliação neurológica para indivíduos com história de tontura ou desmaio durante ou após esforço físico e história de quedas da própria altura, devem ser investigadas. Sabe-se que o treinamento de equilíbrio pode reduzir o risco de queda. ${ }^{4}$

\section{Exercício físico leve}

Para indivíduos que queiram participar de atividade física de leve intensidade, como uma caminhada, o julgamento clínico do médico-assistente deve decidir quais exames prévios devem ser realizados. ${ }^{4}$

Caso seja proposta uma caminhada que não exceda as demandas cardiovasculares das atividades diárias do indivíduo, o teste de esforço não deve ser solicitado rotineiramente. A maioria das diretrizes internacionais evita a solicitação de testes de esforço em indivíduos com baixo risco. ${ }^{5}$

\section{Exercício moderado e vigoroso}

Antes de iniciar um programa de exercício moderado ou vigoroso, o paciente deve ser avaliado em um exame físico rigoroso, com avaliação do controle metabólico em relação à presença de hipoglicemias ou hiperglicemias, limitações físicas, medicações em uso, complicações macro e microvasculares associadas, coagulopatias, avaliação oftalmológica, renal, de membros inferiores e do sistema autonômico. ${ }^{4}$

Algumas patologias podem contraindicar a prática do exercício ou aumentar o risco de lesões, como: hipertensão arterial descontrolada, neuropatia autonômica severa, neuropatia periférica severa com perda de sensibilidade, retinopatia proliferativa. A idade do paciente e seu nível de atividade física prévio também devem ser levados em conta na avaliação clínica.

Todos os aspectos devem ser levados em consideração para que o programa de exercício físico seja eficiente e seguro para o paciente.

Segundo as Diretrizes da Sociedade Brasileira de 


\section{Artigo de revisão}

Diabetes 2013-2014, ${ }^{1}$ devem ser submetidos ao teste de esforço os indivíduos com DM e:

- idade > 35 anos;

- idade > 35 anos e DM1 há mais de 10 anos, ou tipo 2 há mais de 15 anos;

- presença de hipertensão arterial, tabagismo ou dislipidemia;

- suspeita de doenças arterial coronariana, cerebrovascular e/ou arterial periférica;

- neuropatia autonômica;

- nefropatia grave, retinopatia.

Ainda, na ausência de contraindicação, o teste de exercício pode ser realizado em todos os indivíduos com DM para obtenção da frequência cardíaca máxima, da capacidade funcional e para orientação do exercício. ${ }^{1}$

\section{Prescrição do exercício}

\section{Diabetes tipo 1 - Recomendações e prescrição}

Durante a prática de atividade física (AF), em indivíduos não diabéticos, para o ajuste metabólico ser adequado e preservar a normoglicemia, o nível de insulina deve ser o suficiente para permitir a captação muscular de glicose, porém garantir a liberação dos estoques de glicogênio hepático. Esta liberação dos estoques de glicogênio é mediada pelo aumento da produção de adrenalina e glucagon. Contudo, este mecanismo não ocorre no indivíduo com diabetes tipo 1 pela presença de insulina exógena. ${ }^{6}$ Logo, há um risco maior de haver hipoglicemia imediatamente depois da sessão ou horas após a prática de atividade física.

Em seu estudo, Rabasa-Lhoret e colaboradores propuseram um esquema para adaptação de doses de insulina relacionado à duração e à intensidade do exercício quando realizado no período pós-prandial. ${ }^{7}$

Para o exercício planejado, a redução na dose de insulina é o método mais adequado para a redução da hipoglicemia. No entanto, para o exercício não programado, a ingestão adicional de carboidrato (CHO) é necessária. ${ }^{6}$

O paciente com diabetes tipo 1 deve ter seu programa de treinamento individualizado, de acordo com suas necessidades, e ter disponível carboidrato de rápida absorção para que seja consumido em caso de hipoglicemia. ${ }^{6}$

Estas recomendações são úteis para evitar a hipoglicemia, que pode ocorrer durante, imediatamente depois e até mesmo horas após a prática de exercícios. A American Diabetes Association (ADA) sugere orientações gerais que podem ser úteis na regulação da resposta glicêmica ao exercício: ${ }^{8}$

- Evitar a prática de AF se a glicemia estiver $>250 \mathrm{mg} /$ dl e cetose presente. Ser cauteloso com a glicemia > $300 \mathrm{mg} / \mathrm{dl}$, porém sem cetose;

- Controle metabólico antes do exercício;

- Ingerir carboidrato se a glicemia estiver $<100 \mathrm{mg} / \mathrm{dl}$. Pacientes com diabetes tipo 1, sem complicações que impeçam a prática de atividade física e que possuam um bom controle glicêmico, estão aptos a realizar todos os níveis de AF, como lazer, esporte recreacional e profissional. ${ }^{8}$

Dentre uma grande gama de opções de exercício, podemos nos perguntar qual hipoteticamente seria mais benéfico para o paciente diabético. De acordo com o estudo de Yardley e colaboradores, no início da sessão o exercício resistido tem um declínio da glicemia menor que no exercício aeróbio. Porém, o exercício resistido apresenta um efeito de redução da glicemia mais prolongado no pós-exercício. ${ }^{9}$ Sendo assim, seria interessante uma combinação dos dois tipos de exercício, pois apresentam diferentes formas de ação sobre a glicemia, as quais se completam.

De acordo com o American College of Sports Medicine (ACSM), para pacientes com diabetes tipo 1, os exercícios aeróbios devem ser realizados numa frequência de 3-5 vezes por semana, por aproximadamente 20-60 minutos, e sua intensidade deve estar entre 40-85\% do $\mathrm{VO}_{\text {2máx }}$ ou a 55-90\% da frequência cardíaca máxima. ${ }^{10}$ Recomenda-se para os exercícios resistidos a inclusão de pelo menos 8-10 exercícios diferentes envolvendo grandes grupos musculares com a frequência de 2-3 vezes por semana. ${ }^{11}$

\section{Diabetes tipo 2 - Recomendações e prescrição}

O objetivo do tratamento em indivíduos com diabetes tipo 2 é alcançar e manter bom controle glicêmico, do perfil lipídico e de pressão arterial, visando prevenir ou retardar complicações crônicas. ${ }^{3}$ Em um estudo de intervenção composto por uma sessão aguda de exercício aeróbio, resistido e de flexibilidade na mesma sessão em indivíduos com diabetes tipo 2, foi observada melhora nas variáveis glicemia de jejum, HbA1c, lipídios plasmáticos, frequência cardíaca de repouso e índice de massa corporal. ${ }^{12}$ Este estudo também demonstrou o mesmo efeito hipoglicemiante como resposta do exercício físico em pacientes tratados ou não com insulina. Logo, podemos mais uma vez demonstrar a importância do exercício físico para o indivíduo com diabetes tipo $2 .^{12}$

A preocupação com hipoglicemia durante e após 
o exercício não se aplica a pacientes com diabetes tipo 2 que estejam bem controlados, pois os riscos são menores. Assim, uma monitorização da glicemia muito rígida é desnecessária. Porém, aferir a glicemia antes e após o exercício é uma forma de verificar os efeitos do exercício na glicemia e sua eficácia no controle desta. ${ }^{3}$ Além disso, pacientes com diabetes tipo 2 geralmente não necessitam adiar a prática de exercícios se estes se sentirem bem e se a glicemia estiver acima de $300 \mathrm{mg} /$ dl sem cetose. É prudente ser cauteloso e assegurar que o paciente esteja hidratado adequadamente. ${ }^{3}$

Este efeito hipoglicemiante durante o exercício ocorre por meio das contrações musculares, as quais aumentam a captação muscular de glicose. Este mecanismo ocorre mesmo na presença de resistência à insulina. A contração muscular aumenta a quantidade de GLUT4 e a sua translocação para a membrana celular, possibilitando assim a captação de glicose, efeito obtido tanto com exercício aeróbico quanto com exercício resistido. Este aumento da captação de glicose pode se manter elevada pós-exercício por várias horas. ${ }^{3}$

De acordo com o posicionamento em conjunto da ADA e do ACSM (3), o exercício aeróbio em diabéticos tipo 2 deve ser realizado pelo menos três vezes na semana, com uma intensidade moderada que aproximadamente corresponda a $40-60 \%$ do $\mathrm{VO}_{2 \operatorname{máx}}$. Esta intensidade pode ser elevada para vigorosa $\left(>60 \% \mathrm{VO}_{2 \text { máx }}\right)$ se o paciente estiver apto a esta adaptação. Estudos sugerem que pacientes que já se exercitam moderadamente ao intensificar seu treinamento obtêm um ganho adicional dos benefícios do exercício no controle glicêmico. Quanto à duração, é recomendado que o paciente pratique o exercício com intensidade moderada a vigorosa pelo menos 150 minutos por semana. ${ }^{3}$

Já o exercício resistido deve ser realizado pelo menos duas vezes por semana, em dias não consecutivos. Porém, o ideal seria três vezes na semana e sempre combinado com o exercício aeróbio. A intensidade deve ser de moderada ( $50 \%$ de uma repetição máxima) a vigorosa (75-80\% de uma repetição máxima), com o objetivo de ganho de força e melhor ação da insulina. Cada sessão de treinamento deve ser composta de cinco a dez exercícios, os quais devem envolver grandes grupamentos musculares. ${ }^{3}$

Para proporcionar uma melhor aptidão física, é recomendado que os exercícios aeróbios e resistidos fossem combinados na sessão de treinamento. Essa integração permite que os benefícios do exercício sejam completos, ou seja, além do controle metabólico do diabetes, a prática também beneficia a capacidade cardiorrespiratória, e o aumento da força e da resistência muscular, essenciais para uma melhor qualidade de vida. ${ }^{13}$

Após atividade física de treinamento resistido, o consumo de carboidrato de rápida absorção somado ao consumo de proteína isolada do soro do leite e de leucina parecem levar a uma maior síntese proteica quando comparado ao consumo de carboidrato isoladamente. ${ }^{14}$

\section{Conclusão}

Com base na revisão de literatura, a prescrição de exercício físico é um meio eficaz de corroborar com o tratamento medicamentoso e nutricional no controle metabólico do diabetes. Contudo, antes dos indivíduos com diabetes iniciarem qualquer programa de exercícios, estes devem ser avaliados pelo médico minuciosamente. Isto é fundamental para uma prática segura.

A prescrição de exercício deve ser realizada por profissional capacitado, respeitando algumas recomendações em relação ao controle glicêmico antes e depois do exercício, visando à segurança e ao acompanhamento de seu benefício. Estando bem controlados e na ausência de contraindicações, pacientes com diabetes podem realizar diversas modalidades de exercícios. Tanto atividades aeróbias quanto os exercícios resistidos oferecem benefícios metabólicos. Logo, o exercício deve ser estimulado visando ao controle glicêmico, à saúde e à qualidade de vida.

\section{Referências}

1. Sociedade Brasileira de Diabetes. Diretrizes da Sociedade Brasileira de Diabetes 2013-2014; 2014 [acesso em 2015 nov 22]. Disponível em: http://www.nutritotal.com.br/diretrizes/ files/342--diretrizessbd.pdf

2. Nielsen PJ, Hafdahl AR, Conn VS, et al. Meta-analysis of the effect of exercise interventions on fitness outcomes among adults with type 1 and type 2 diabetes. Diabetes Res Clin Pract. 2006 Nov;74(2):111-20.

3. Colberg SR, Sigal RJ, Fernhall B, et al. Exercise and type 2 diabetes: the American College of Sports Medicine and the American Diabetes Association: joint position statement. Diabetes Care. 2010 Dec;33(12):e147-67.

4. Morrison S, Colberg SR, Mariano M, et al. Balance training reduces falls risk in older individuals with type 2 diabetes. Diabetes Care. 2010 Apr;33(4):748-50.

5. Colberg SR, Sigal RJ. Prescribing exercise for individuals with type 2 diabetes: recommendations and precautions. Phys Sportsmed. 2011 May;39(2):13-26.

6. Ramalho AC, Soares S. [The role of exercise in the treatment of type 1 diabetes]. Arq Bras Endocrinol Metabol. 2008 Mar;52(2):260-7.

7. Rabasa-Lhoret R, Bourque J, Ducros F, et al. Guidelines for premeal insulin dose reduction for postprandial exercise of different intensities and durations in type 1 diabetic subjects treated intensively with a basal-bolus insulin regimen (ul- 


\section{Artigo de revisão}

tralente-lispro). Diabetes Care. 2001 Apr;24(4):625-30.

8. Physical activity/exercise and diabetes. Diabetes Care. 2004 Jan;27 Suppl 1:S58-62.

9. Yardley JE, Kenny GP, Perkins BA, et al. Resistance versus aerobic exercise: acute effects on glycemia in type 1 diabetes. Diabetes Care. 2013 Mar;36(3):537-42.

10. Medicine ACoS. Diretrizes da ACSM para os testes de esforço e sua prescrição. Rio de Janeiro: Guanabara Koogan; 2011.

11. Colberg SR. Use of clinical practice recommendations for exercise by individuals with type 1 diabetes. Diabetes Educ. 2000 Mar-Apr;26(2):265-71.

12. Silva CA, Lima WC. Efeito benéfico do exercício físico no con- trole metabólico do diabetes mellitus tipo 2 a curto prazo. Arq Bras Endocrinol Metabol. 2002;46(5):550-6.

13. Arsa G, Lima L, Almeida SS, et al. Type 2 Diabetes Mellitus:Physiological and genetic aspects and the use of physical exercise for diabetes control. Rev Bras Cineantropom Desempenho Hum. 2009;11:103-11.

14. Koopman R, Wagenmakers AJ, Manders RJ, et al. Combined ingestion of protein and free leucine with carbohydrate increases postexercise muscle protein synthesis in vivo in male subjects. Am J Physiol Endocrinol Metab. 2005 Apr;288(4):E645-53. 